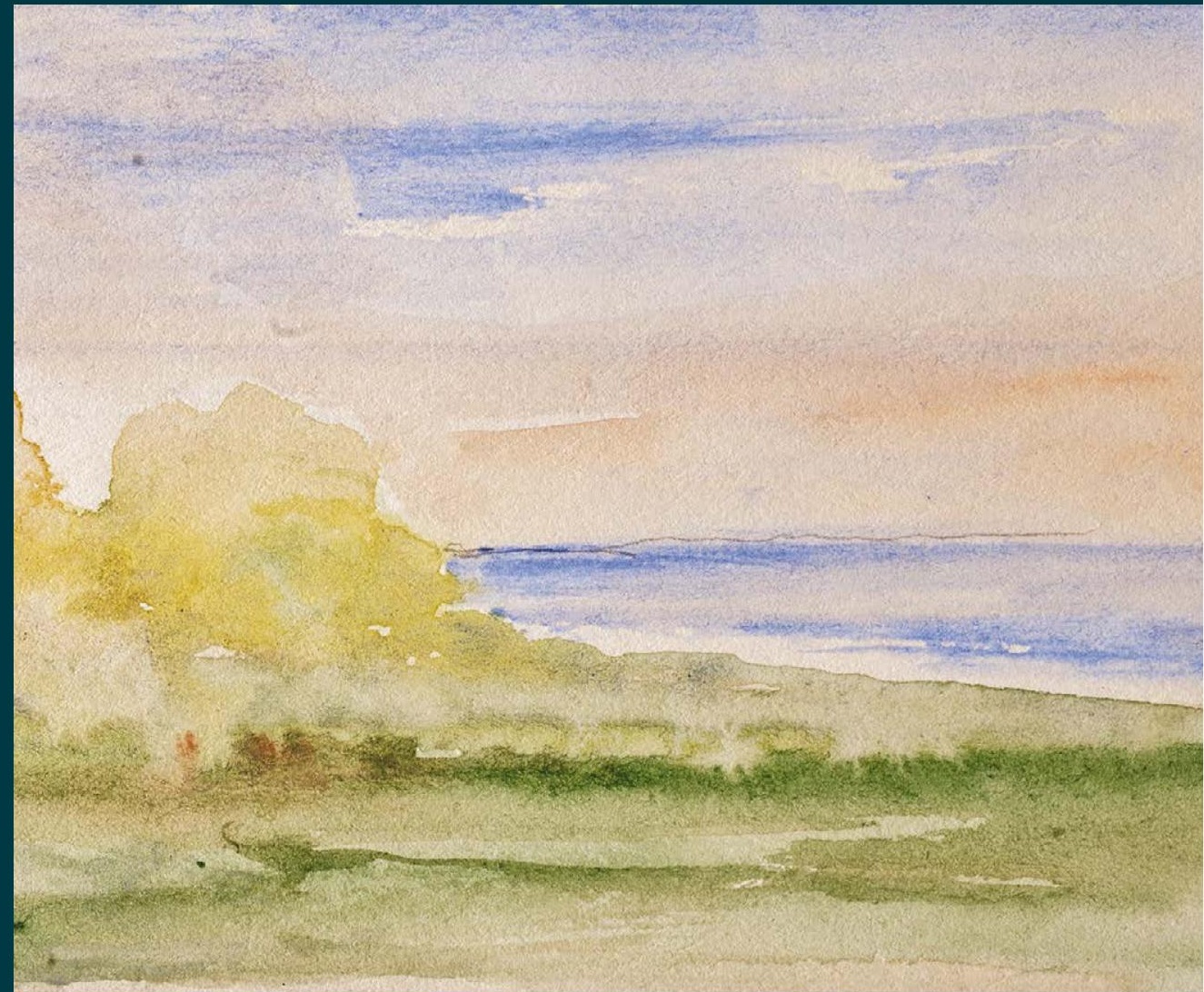

Il pensiero critico fra geografia e scienza del territorio

Scritti su Massimo Quaini

a cura di

Roberta Cevasco, Carlo Alberto Gemignani, Daniela Poli, Luisa Rossi

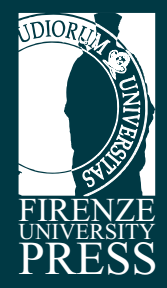


TERRITORI

ISSN 2704-5978 (PRINT) | ISSN 2704-579X (ONLINE)

$-33-$ 


\section{DIRECTOR}

Daniela Poli, University of Florence, Italy

\section{SCIENTIFIC BOARD}

Iacopo Bernetti, University of Florence, Italy Leonardo Chiesi, University of Florence, Italy Claudio Fagarazzi, University of Florence, Italy David Fanfani, University of Florence, Italy Fabio Lucchesi, University of Florence, Italy Alberto Magnaghi, University of Florence, Italy Carlo Natali, University of Florence, Italy Gabriele Paolinelli, University of Florence, Italy Camilla Perrone, University of Florence, Italy Claudio Saragosa, University of Florence, Italy

\section{INTERNATIONAL SCIENTIFIC BOARD}

Paolo Baldeschi, University of Florence, Italy

Luisa Bonesio, University of Pavia, Italy

Lucia Carle, EHESS, School of Advanced Studies in the Social Sciences, France

Pier Luigi Cervellati, University of Venice Iuav, Italy

Giuseppe Dematteis, Politecnico di Torino, Italy

Pierre Donadieu, ENSP, National School of Landscape Architecture, France

Giorgio Ferraresi, Politecnico di Milano, Italy

André Fleury, ENSP, National School of Landscape Architecture, France

Carlo Alberto Garzonio, University of Florence, Italy

Rossano Pazzagli, University of Molise, Italy

Bernardino Romano, University of L'Aquila, Italy

Leonardo Rombai, University of Florence, Italy

Bernardo Rossi-Doria, University of Palermo, Italy

Wolfgang Sachs, Wuppertal Institute, Germany

Bruno Vecchio, University of Florence, Italy

Sophie Watson, The Open University, United Kingdom

\section{MANAGING EDITOR}

Angelo Maria Cirasino, University of Florence, Italy

La collana Territori nasce nel 2007 per iniziativa di ricercatori e docenti dei Corsi di laurea interdipartimentali in Pianificazione dell'Università di Firenze, Dipartimenti di Architettura (DiDA), Agraria (DAgri) e Ingegneria civile (DICEA). Il Corso di laurea triennale (Pianificazione della città, del territorio e del paesaggio) e quello magistrale (Pianificazione e progettazione della città e del territorio) hanno sviluppato in senso multidisciplinare i temi del governo e del progetto del territorio messi a punto dalla "scuola territorialista italiana". Tale approccio ha assegnato alla didattica un ruolo centrale nella formazione di figure professionali qualificate nella redazione e nella gestione di strumenti ordinativi del territorio, in cui i temi dell'identità, dell'ambiente, del paesaggio, dell'empowerment sociale, dello sviluppo locale rappresentano le componenti più rilevanti. La collana Territori continua quest'opera sul versante editoriale promuovendo documenti di varia natura (saggi, ricerche, progetti, seminari, convegni, tesi di laurea, didattica) che sviluppano questi temi, accogliendo proposte provenienti da settori nazionali e internazionali della ricerca. 


\section{Il pensiero critico fra geografia e scienza del territorio}

Scritti su Massimo Quaini

a cura di

\section{Roberta Cevasco, Carlo Alberto Gemignani, Daniela Poli, Luisa Rossi}

scritti di

Filippo Celata, Roberta Cevasco, Annalisa D'Ascenzo, Elena Dai Prà, Valeria De Marcos, Valentina De Santi, Giuseppe Dematteis, Nicola Gabellieri, Carlo A. Gemignani, Claudio Greppi, Anna Guarducci, Alberto Magnaghi, Giorgio Mangani, Anna Marson, Carla Masetti, Diego Moreno, Alessandro Panetta, Valentina Pescini, Daniela Poli, Paola Pressenda, Leonardo Rombai, Luisa Rossi, Massimo Rossi, Anna Maria Stagno, Maria Luisa Sturani, Francesco Surdich, Marcello Tanca, Vittorio Tigrino, Francesco Vallerani

Firenze University Press 
Il pensiero critico fra geografia e scienza del territorio : scritti su Massimo Quaini / a cura di Roberta Cevasco, Carlo Alberto Gemignani, Daniela Poli, Luisa Rossi. - Firenze : Firenze University Press, 2021.

(Territori ; 33)

https://www.fupress.com/isbn/9788855183222

ISSN 2704-5978 (print)

ISSN 2704-579X (online)

ISBN 978-88-5518-321-5 (print)

ISBN 978-88-5518-322-2 (PDF)

ISBN 978-88-5518-323-9 (XML)

DOI $10.36253 / 978-88-5518-322-2$

Graphic design: Alberto Pizarro Fernández, Lettera Meccanica srl.

Cura redazionale, editing testi e immagini, ottimizzazione grafica, postediting e impaginazione: Angelo M. Cirasino.

Front cover: Pompeo Mariani (1857-1827), Marina, 1875, collezione privata. Acquarello su carta, 9x16.

Questo volume è stato edito grazie al contributo: degli autori; delle Università di Cagliari (DSBCT), Firenze (DiDA), Parma (DUSIC), Roma "La Sapienza" (MeMoTEF), Torino (DSS) e IUAV di Venezia (DCP); della Società dei Territorialisti e delle Territorialiste ONLUS.

FUP Best Practice in Scholarly Publishing (DOI https://doi.org/10.36253/fup_best_practice) All publications are submitted to an external refereeing process under the responsibility of the FUP Editorial Board and the Scientific Boards of the series. The works published are evaluated and approved by the Editorial Board of the publishing house, and must be compliant with the Peer review policy, the Open Access, Copyright and Licensing policy and the Publication Ethics and Complaint policy.

Firenze University Press Editorial Board

M. Garzaniti (Editor-in-Chief), M.E. Alberti, F. Arrigoni, M. Boddi, R. Casalbuoni, F. Ciampi, A. Dolfi, R. Ferrise, P. Guarnieri, A. Lambertini, R. Lanfredini, P. Lo Nostro, G. Mari, A. Mariani, P.M. Mariano, S. Marinai, R. Minuti, P. Nanni, A. Novelli, A. Orlandi, A. Perulli, G. Pratesi, O. Roselli.

¿ The online digital edition is published in Open Access on www.fupress.com.

Content license: the present work is released under Creative Commons Attribution 4.0 International license (CC BY 4.0: http://creativecommons.org/licenses/by/4.0/legalcode). This license allows you to share any part of the work by any means and format, modify it for any purpose, including commercial, as long as appropriate credit is given to the author, any changes made to the work are indicated and a URL link is provided to the license.

Metadata license: all the metadata are released under the Public Domain Dedication license (CCO 1.0 Universal: https://creativecommons.org/publicdomain/zero/1.0/legalcode).

(C) 2021 Author(s)

Published by Firenze University Press

Firenze University Press

Università degli Studi di Firenze

via Cittadella, 7, 50144 Firenze, Italy

www.fupress.com

This book is printed on acid-free paper

Printed in Italy 


\section{Sommario}

Premesse

Daniela Poli $\quad$ IX

Roberta Cevasco, Carlo A. Gemignani, Daniela Poli, Luisa Rossi XI

\section{Massimo Quaini geografo critico}

L'Alto e il Basso

Giorgio Mangani

\section{Geografie}

Intorno a Geografia Democratica. Fra Marx e Foucault, I'Italia e I"Ammerica'

Filippo Celata

Massimo Quaini e la geografia di avanguardia: dal Marxismo e geografia all'Anarchismo e geografia

Valeria De Marcos

Massimo Quaini: geografia storica, fonti, conoscenze territoriali e loro uso politico e socio-culturale

Leonardo Rombai

II ruolo dell'utopia, del mito e dell'immaginario nella concezione della geografia di Massimo Quaini

Francesco Surdich

Una lettura dell'approccio quainiano alla cartografia attraverso la lente degli ingegneri geografi napoleonici

Valentina De Santi

\section{Paesaggio e territorio}

Una dottrina rivoluzionaria della sistemazione dello spazio. Massimo Quaini geografo-pianificatore

Giuseppe Dematteis

Massimo Quaini, territorialista

Alberto Magnaghi

FUP Best Practice in Scholarly Publishing (DOI 10.36253/fup_best_practice)

Roberta Cevasco, Carlo Alberto Gemignani, Daniela Poli, Luisa Rossi (edited by), II pensiero critico fra geografia e scienza del territorio. Scritti su Massimo Quaini, (c) 2021 Author(s), content CC BY 4.0 International, metadata CC0 1.0 Universal, published by Firenze University Press (www.fupress.com), ISSN 2704-579X (online), ISBN 978-88-5518-322-2 (PDF), DOI 10.36253/978-88-5518-322-2 
Predoni a casa nostra e il geografo solidale: Massimo Quaini tra terra e acqua

Francesco Vallerani

Massimo Quaini, studioso del paesaggio

Anna Marson

Paesaggio e musei: sulle tracce di Massimo Quaini

Paola Pressenda, Maria Luisa Sturani

Massimo Quaini, la passione per la cartografia storica con uno sguardo rivolto al futuro

Daniela Poli

Massimo Quaini, bricoleur. Su un libro che avrebbe potuto essere e non fu

Marcello Tanca

\section{Esperienze di ricerca}

Massimo Quaini: la Liguria labirinto e laboratorio

Carlo A. Gemignani

Sulla geograficità della ecologia storica: contributi di Massimo Quaini

Roberta Cevasco, Diego Moreno

Lo sguardo del geografo: Massimo Quaini, I'archeologia, la storia

Anna Stagno, Vittorio Tigrino

Dalla geografia storica all'archeologia del paesaggio e dell'ambiente. Una irrinunciabile eredità di Massimo Quaini

Alessandro Panetta, Valentina Pescini

Massimo Quaini e il viaggio: il ruolo della verticalità

Claudio Greppi

Il filo da riannodare: Massimo Quaini, una letteratura per la geografia e una geografia per la letteratura

Nicola Gabellieri

\section{Contributi per una biografia}

Massimo Quaini e il CISGE

Annalisa D'Ascenzo, Elena Dai Prà, Anna Guarducci, Carla Masetti, Massimo Rossi

Raccontare, raccontarsi. Massimo Quaini fra biografia ed 'egogeografia'

Luisa Rossi

Appendice

Massimo Quaini. Bibliografia 1963-2020

a cura di Valentina De Santi 


\title{
Il filo da riannodare: Massimo Quaini, una letteratura per la geografia e una geografia per la letteratura
}

Nicola Gabellieri

\begin{abstract}
A proposito del rapporto tra opere letterarie e studi geografici, l'interesse di Massimo Quaini si è consolidato in una impronta euristica la cui originalità non è stata ancora adeguatamente sottolineata. Attraverso l'esegesi dei suoi saggi, il contributo identifica alcuni punti chiave del suo pensiero: metodologicamente, la necessità di un approccio critico di 'archeologia dei saperi e delle fonti' al profilo dello scrittore; teleologicamente, l'identificazione dei fattori geografici interni alle opere come medium valorizzativo del territorio; 3 . epistemologicamente, l'utilizzo di topoi e metafore letterarie come chiave interpretativa - a corollario delle analisi geografico-storiche - delle dinamiche territoriali storiche e attuali.
\end{abstract}

Keywords: Massimo Quaini; geografia letteraria; geografia storica; valorizzazione territoriale; saperi geografici.

\section{Premessa}

Recentemente, il complesso di studi che, con varie metodologie e obiettivi, si può raccogliere sotto la macrocategoria di 'geografie letterarie', ha conosciuto una crescente fortuna in ambito italiano e globale (Brosseau 2017). Se la relazione tra la letteratura come strumento euristico e l'analisi dei fenomeni spaziali può essere rintracciata sin dagli albori della geografia come scienza moderna, i primi tentativi di sistematizzazione di questa fonte sono databili agli anni '70 (GAvinELLi 2007; LÉvy 2006; SCARAMEllini 1985). In questa "storia infinita di incontri e scambi reciproci” (MARENGo 2016, 13), a Massimo Quaini è stato riconosciuto un ruolo di precursore per la tradizione italiana. Eppure, scorrendo la sua ricca e articolata produzione bibliografica si può notare come il geografo ligure non abbia prodotto, con poche eccezioni (QUAINI 1995; 2009c; 2016), contributi esplicitamente dedicati ad analisi storico-strutturaliste o culturaliste di racconti o romanzi, 
nonostante i frequentissimi rimandi a brani e immagini letterari presenti nei suoi scritti. ${ }^{1}$

Tradizionalmente, il rapporto di lunga durata tra geografia e letteratura viene suddiviso in due diverse prospettive: da un lato una posizione strutturalista, più propria di un approccio storicista e umanistico, che ha affrontato opere come i resoconti di viaggio o la letteratura regionale quali fonti di documentazione veristica per la ricostruzione di paesaggi, territori e pratiche del passato; dall'altro, un fronte di ispirazione più culturalista si è invece dedicato ad esplorare i testi letterari quali prodotti di percezioni e processi cognitivi, indugiando quindi nella loro dimensione di soggettività culturale e individuale nella rappresentazione della realtà spaziale (Scaramellini 1985; Lando 1993; Persi, Dai Prà 2001; FonNesu, Rombai 2004; LÉvy 2006; GabelLieri 2019, 11-23).

Alla luce di questa dicotomia classica, il presente contributo mira invece a identificare il rapporto di Massimo Quaini con la fonte letteraria come una 'terza via', dalle caratteristiche complesse e molteplici, attraverso la quale il suo interesse per la narrativa e la poetica si è consolidato in una impronta epistemologica e metodologica dai tratti peculiari, la cui originalità non è stata ancora adeguatamente sottolineata.

Spigolando nella vasta produzione saggistica di Quaini, questo contributo si propone di presentare vari spunti di riflessione-

1 Oltre al conterraneo Italo Calvino, onnipresente nella produzione saggistica quainiana degli ultimi venti anni (QUAINI 1995; 2002; 2006a; 2006b; 2006c; 2008; 2009a; 2009c; 2010; 2011, 2013; 2014a; 2016a), si possono menzionare Ludovico Ariosto (2002), Honoré de Balzac (ibidem), Tahar Ben Jelloun (ibidem), Francesco Biamonti (ibidem; 2006a; 2006b; 2013), Giovanni Boine (2006a), Jorge Luis Borges (1994; 2002; 2003, 2006a; 2006b; 2010), Albert Camus (2002; 2016b), Elias Canetti (2014a), Giorgio Caproni (2006a; 2006b), Lewis Carroll (2002; 2006a), Guido Ceronetti (ibidem), Samuel Coleridge (2002), Gustave Flaubert (ibidem), Jean Giono (2016), Johann Wolfgang von Goethe (2002; 2006a; 2006c; 2009; 2016b), Victor Hugo (1996; 2002), Franz Kafka (ibidem; 2006a; 2015), Milan Kundera (2002), Carlo Levi (2011), Alessandro Manzoni (2015), Guy de Maupassant (2006b), Montaigne (2006c), Eugenio Montale (2002; 2006a), Robert Musil (2002; 2006a), Anna Maria Ortese (ibidem), Pier Paolo Pasolini (ibidem), Cesare Pavese (ibidem), Marcel Proust (2002), Raymond Queneau (1994), Antoine de Saint-Exupéry (2002), Josè Saramago (2006a), Camillo Sbarbaro (ibidem; 2006b), Leonardo Sciascia (2009b; 2010), Mario Soldati (2002; 2006a), Stendhal (1996), Jonathan Swift (2002), Paul Valéry (ibidem; 2006a, 2016a), Jules Verne (1995), Oscar Wilde (2002; 2006a), Virginia Woolf (2002). 
senza pretesa di offrire una trattazione esaustiva - su questa intersezione tra diverse discipline secondo il pensiero 'geograficoletterario' quainiano. In questa sede si è scelto di affrontare la bibliografia del geografo ligure come un corpus unitario, facendo ampio riferimento a brani provenienti da diversi saggi per identificare una serie di punti chiave aggregati in tre diverse tematiche: epistemologicamente, l'utilizzo di topoi letterari e metafore come chiave interpretativa - a corollario delle analisi geografico-storiche - di ben delineate dinamiche sociali e territoriali storiche ed attuali; metodologicamente, la necessità di un approccio critico al profilo e al contesto dello scrittore, per identificare i saperi che stanno alla base della sua narrazione dello spazio; teleologicamente, l'identificazione e l'analisi degli elementi geografici interni alle opere come medium di valorizzazione del territorio, necessariamente integrati con un adeguato sviluppo del comparto produttivo rurale.

\section{La letteratura come chiave interpretativa}

"Nel corso della mia esplorazione dell'universo ligure sono stato conquistato da un'oscura e appunto labirintica valletta [...] che porta il nome di Gambatiggia. Da essa proviene il manoscritto che ora raccomando alle tue amorevoli cure" (QuAINi 2002, 10). È con questo classico topos della letteratura ottocentesca - il manoscritto ritrovato casualmente, usato tra gli altri da Manzoni e Potocki - che Quaini introduce la sua monografia dalla foggia più originale, La mongolfiera di Humboldt. L'uso di artifizi narrativi ispirati dalla letteratura è uno dei leitmotiv dei saggi quainiani redatti almeno a partire dagli anni '90; i suoi testi dedicati alla storia del paesaggio, alle geografie territoriali e alla storia della cartografia sono costellati di citazioni di brani di autori più o meno noti, a testimonianza della vasta conoscenza del geografo ligure e del suo amore per la scrittura. Tali citazioni sono indirizzate, perlopiù, ad illustrare in chiave metaforica ed esemplificativa alcuni nodi teorici e metodologici del suo pensiero; è il caso, ad esempio, di quando Quaini distingue le scale di indagine macrostorica e microstorica, paragonando la prima allo stile narrativo di Hugo e la seconda a quello di Stendhal (QuAini 1996, 9-10), o della sua comparazione tra le criticità della attuale geografia accademica e l'irraggiungibile castello tratteggiato da Kafka (QuAini 2015). 
Sarebbe però riduttivo limitare il valore di queste citazioni ad una mera, per quanto apprezzabile, funzione estetica, o ad intenti puramente esemplificativi. Più propriamente, è possibile osservare come in ottica quainiana le forti e suggestive immagini mutuate dalla letteratura assumano un valore di allegoria euristica. Scrive infatti Quaini che tutta la scienza, compresa quella geografica,

non può fare a meno dell'analogia e della metafora; [...] le analogie non superficiali aprono la pista di nuove interpretazioni o spiegazioni, obbligando a cercare e definire la logica interna del fenomeno che viene improvvisamente illuminato dalla metafora. La scienza ha avuto bisogno e continua ad aver bisogno delle metafore più ardite (QuAINi 1995, 59).

Gran parte degli studi di Quaini sono stati dedicati alla 'sua' Liguria. Ed è proprio illustrando la regione mediterranea che egli sottolinea:

mi sono convinto che in Liguria i testimoni e gli osservatori più lucidi, anche per quanto riguarda le ferite, le lacerazioni del paesaggio (la speculazione edilizia, lo spopolamento montano) sono stati gli scrittori, i poeti. Mi sono convinto di quanta ragione avesse Musil nell'attribuire un carattere razionale e innovativo alla conoscenza del poeta (QuAini 2006b, 10).

Non è un caso, infatti, che molti dei nodi di riflessione sui fenomeni geografici storici e attuali dei paesaggi mediterranei sollevati da Quaini siano in parte mutuati da ricorrenti topoi narrativi dei suoi autori più amati: si pensi alla figura del passeur biamontiano, espressione di confini fisici o politici che nella pratica delle popolazioni si rivelano porosi; ai processi di riqualificazione degli spazi rurali litoranei; alla divisione tra abrigu e ubagu suggerita da Calvino, cioè tra Liguria urbana e rivierasca e Liguria interna, entrambe facce interrelate della stessa medaglia; o alla lettura del paesaggio rurale come 'luogo di produzione' ispirata anche dai romanzi di Biamonti e Boine. ${ }^{2}$

${ }^{2}$ Funzioni, quella degli scrittori come produttori di idee e interpretazioni, e quella delle fonti letterarie come allegorie euristiche, annotate anche nelle riflessioni di LANDo 1993 e impiegate con fini euristici da Gavinelli, Zanolin 2019. 
La capacità di sviluppare nuove teorie interpretative sui processi storico-territoriali viene riconosciuta all'occhio sensibile del letterato soprattutto per un oggetto geografico ben determinato, il paesaggio. Data la natura ambigua inerente a questo concetto fluido - "che non ha la materialità del territorio o dell'ambiente, in quanto invenzione storica ed essenzialmente estetica" (assunto parafrasato da Kafka in QuAIn 2006a, 13), quindi al tempo stesso invenzione estetica e prodotto storico - Quaini esalta la capacità esegetica e interpretativa dei paesaggi materiali rappresentata dalla mediazione artistica, che rimane un fil rouge di molti dei suoi più tardi saggi: e quindi "per costruire le nuove mappe e alimentare i nostri progetti sui paesaggi di Liguria dobbiamo seguire il percorso tracciato dallo scrittore ligure più lucido al quale ci siamo già ripetutamente richiamati, Italo Calvino, senza tirarlo troppo da una parte o dall'altra" (ivi, 170).

Ridefinendo quindi il ruolo degli intellettuali - scrittori, ma anche pittori - quali osservatori privilegiati, Quaini riconosce alla loro testimonianza la capacità di individuare l'identità paesistica regionale, aiutando a comporre una lettura capace di collegare la materialità del paesaggio (inteso come strutture agrarie, insediamenti, gestione delle risorse silvo-pastorali, usi del suolo, ecc.) con il significato ad esso attribuito dalle varie società (ivi, 13), quindi tale da poter fornire al geografo-scienziato chiavi interpretative efficaci.

\section{Strategie metodologiche per le fonti letterarie}

Illustrando nel 1981 l'origine e lo stato della geografia storica italiana, Paola Sereno riconosce a Quaini il merito di aver cercato "di costruire una metodologia geo-storica [sperimentando] la sua metodologia nell'analisi delle strutture agrarie della Liguria medievale e moderna, dove ricevono adeguata illustrazione pratica i suoi concetti di analisi integrata dei livelli spazio-temporali e storico-ecologici" (SERENo 1981, 169; 174), recependo e sviluppando sia i metodi di esegesi e analisi filologica della fonte storica sviluppati dalla scuola delle Annales sia l'uso integrato di fonti multiple documentarie e di terreno di ispirazione anglosassone. 
Quale corollario alla teoria metodologica quainiana, che presuppone il più ampio ventaglio di documenti, anche ai testi letterari viene infatti riconosciuto il valore di fonte storica. Nonostante l'approccio al paesaggio rurale di scrittori e poeti sia connotato da forti aspetti archetipici e simbolici, ciò non rende impossibile la lettura di questi volumi anche con occhi documentali, nonostante la necessità di alcune precauzioni metodologiche: nell'introduzione ad un volume esplicitamente dedicato alla geografia letteraria, Quaini $(2011,9)$ tende ad ammonire contro una lettura puramente simbolica dell'opera letteraria: "è stato proprio Calvino a insegnarci che questa vocazione letteraria della geografia può essere insufficiente e pericolosa se viene intesa secondo un modello di letteratura che privilegia più la finzione che il fatto". Pioniere negli studi di approccio strutturalista, volti ad indagare i processi di territorializzazione dello spazio materiale nella loro dimensione storica di continuità e discontinuità, Quaini, seppur aprendosi negli anni seguenti allo studio delle identità territoriali anche attraverso l'analisi delle opere culturali, non ha mai mancato di fustigare "la cosiddetta geografia culturale postmoderna [...] che significa rinchiudere la geografia entro l'esclusivo orizzonte delle rappresentazioni simboliche [...] con il risultato finale di escludere qualsiasi possibilità di decifrazione analitica o realistica del paesaggio" (QUAINI 2006a, 91).

La valenza della letteratura come fonte euristica geografico-storica è quindi attribuita soprattutto ai testi odeporici o agli scrittori realisti e locali - intendendo con questa accezione coloro che hanno una forte e documentata conoscenza del territorio che descrivono come quelli che hanno contraddistinto il filone regionale ligure neorealista; coloro che, come Calvino, Caproni, Montale o Biamonti sono stati capaci di adottare "uno sguardo a 360 gradi" (ivi, 22, mutuando, appunto, una allegoria di Calvino). Eppure, raramente egli sceglie di utilizzare le descrizioni di letterati come indizi per la ricostruzione di pratiche o di strutture paesaggistico-territoriali, contrariamente al modello adottato ad esempio da Iolanda Fonnesu e Leonardo Rombai (2004) per la Toscana. Ciononostante, Quaini non manca di riflettere sulle opportune strategie metodologiche da assumere per approcciare un documento letterario. Le parole di apprezzamento che Quaini rivolge a Biamonti - "è soprattutto lo sguardo, la capacità di cogliere i più piccoli dettagli, le anomalie e specificità locali, a fornire a Biamonti la materia dei romanzi. 
Una capacità di lettura analitica dei paesaggi locali, individuali, che non perde mai di vista il contesto generale della globalizzazione del mondo" (QuAINi 2016a, 60) - sottolineano l'importanza di una prospettiva capace di combinare uno sguardo fine microanalitico con una generalizzazione positiva per il corretto metodo di analisi geografica. Nello stesso saggio, l'autore invoca la necessità di praticare una "archeologia delle fonti che alimentarono e diedero forza alla straordinaria testimonianza di Biamonti" (ivi, 64), ovvero di adottare un metodo di analisi filologico-storica che, sulla base di documenti di archivio collaterali, sia in grado di costruire una biografia dell'autore e individuare i suoi obiettivi, saperi e conoscenze; richiamando quindi pedissequamente il criterio di ricerca ampiamente sviluppato nei suoi studi sui cartografi liguri (QUAINI 2007). L'uso della letteratura come strumento euristico per la caratterizzazione storico-ambientale del paesaggio non può quindi prescindere dalle tre strategie metodologiche postulate dalla scuola genovese: l'incrocio tra serie di fonti diverse, la critica filologica della fonte e l'approccio diacronico multiscalare (MORENO ET AL. 2005).

\section{Geografia letteraria e applicazione territoriale}

Il problema della applicazione degli studi geografici è un tema a cui Quaini ha sempre dedicato grande rilevanza, richiamando più volte la necessità di una ricaduta delle ricerche geografico-storiche sul paesaggio anche attraverso apposite strutture, come gli 'osservatori' o i 'centri di interpretazione', quali anelli di congiunzione tra ricerca accademica e governance partecipativa del patrimonio storico-ambientale (inter alia, QuAini 2014a; Moreno, Quaini 2016).

Frequentemente Quaini ha criticato le attuali politiche di gestione territoriale liguri, sia nelle loro tendenze di patrimonializzazione, con il rischio di trasformare il Parco Nazionale delle Cinque Terre "in un parco tematico stile Epcot e Disneyland" (QuAini 2006a, 62), sia nelle inefficaci, se non dannose, iniziative di rinaturalizzazione. Già nel 1973 aveva messo in luce la natura altamente complessa e storica dei sistemi agro-silvo-pastorali liguri; e gli spunti forniti dalla letteratura lo rafforzano nella sua ricostruzione dell'entroterra mediterraneo come prodotto di attivazione di risorse ambientali con pratiche e conoscenze locali. 
Ciò premesso, aveva più volte denunciato il rischio di un aumento della fragilità idro-geologica insito nell'abbandonare un territorio plasmato storicamente, auspicando invece una "ripresa produttiva" per favorire una corretta conservazione (QUAINI 2015a, 210).

Nel 2015, un progetto congiunto tra Laboratorio di Archeologia e Storia Ambientale (LASA) dell'Università di Genova, Fondazione Compagnia di San Paolo e Comune di San Biagio della Cima (IM) volto alla creazione di un Parco letterario dedicato a Francesco Biamonti è stato per Quaini l'occasione di applicare alla progettualità territoriale le proprie conoscenze su uno degli autori da lui più amati (Moreno ET AL. 2016).

Il parco letterario designa un organismo nato con il preciso obiettivo di agevolare la valorizzazione di paesaggi noti e meno noti attraverso gli occhi degli autori che vi hanno vissuto e vi hanno trovato ispirazione. A partire dagli anni '90, si è ormai affermato il paradigma di parchi culturali non solo limitati alla salvaguardia del patrimonio, ma come gestori di risorse per promuovere sia lo sviluppo culturale-sociale sia quello economico dei territori celebrati dagli scrittori (Anglani 2000; Persi, Dai PRÀ 2001).

Già nel 2006, Quaini riconosceva alla narrativa di Biamonti la capacità di mostrare la contraddizione inerente al concetto di paesaggio, "fra l'eredità del paesaggio che come immagine è stato costruito da poeti, pittori, scrittori e granturisti e i discendenti di coloro che quel territorio, quell'ambiente hanno abitato e costruito ma che non hanno o poco hanno sentito come paesaggio" (QuAINi 2006b, 10). Esplicitando come i fruitori ultimi di un percorso di valorizzazione culturale debbano essere sia i turisti sia gli abitanti, l'ambiziosa proposta presentata è stata fare del territorio di San Biagio un "parco produttivo", rielaborazione del concetto di "parco agricolo" maturato a partire dagli anni '90 (Fanfani, Magnaghi 2010). Nel caso di San Biagio, per rilanciare le produzioni rurali locali - che sono le pratiche che hanno contribuito a creare il paesaggio narrato da Biamonti - i brani dei suoi testi letterari e i risultati di una serie di ricerche archivistiche e archeologiche sono stati indirizzati a promuovere le attività aziendali agricole storiche in un tentativo di potenziamento produttivo che garantisca anche tutela ambientale. 
Tale progetto ha mostrato quindi come geografia storica, geografia letteraria e programmazione territoriale possano unirsi dialogando su temi comuni, come il paesaggio rurale, i prodotti e i produttori, per perseguire una nuova forma di organizzazione sostenibile dello spazio come incentivo allo sviluppo e come progetto di "tutela produttiva del territorio" (QuAINI 2014b).

\section{Qualche riflessione sulla contaminazione tra geografia e letteratura, tra passato e futuro}

Ci sono dei passi dei suoi scritti in cui Quaini attribuisce agli scrittori liguri l'intero merito del suo percorso di ricerca: "si deve alla sensibilità dei poeti l'elogio della provincia ligure che oggi possiamo riscoprire in prose che fino a ieri abbiamo considerato marginali” (QUAINI 2006a, 34); e proprio grazie a queste prose, che hanno aperto i suoi occhi sulla ricchezza dei saperi e sull'importanza del patrimonio culturale e ambientale delle aree interne, il Nostro ritiene di aver abbandonato lo studio degli spazi urbani, più naturale per un giovane geografo marxista, per spingersi sul meno conosciuto ambito del contesto rurale, l'ubagu.

La preferenza accordata a scrittori 'locali' è paradigmatica di un pilastro del metodo da lui sempre applicato, mutuato in parte dalla scuola microstorica italiana: ovvero che solo da una approfondita conoscenza del territorio è possibile arrivare a una concreta esegesi analitica dei fenomeni sociali. Questa strategia induttiva, che parte da dati concreti per costruire teorie interpretative generali, risulta "basata su un punto di cui Giono, Valéry, Camus e Biamonti avevano piena consapevolezza: che il rapporto locale-globale va rovesciato e che occorre riconoscere e valorizzare non le cause universali che producono effetti locali, ma le cause locali che producono effetti universali" (QUAINi 2016c, 58)

$\mathrm{Ci}$ sono due saggi 'minori' che aiutano a comprendere il valore attribuito alla letteratura come strumento epistemologico: il primo (QuAINi 1995) è dedicato ai contenuti geografici dei romanzi di Jules Verne, l'altro (QUAINI 2014c) alle rappresentazioni cartografiche dei topografi francesi, ma entrambi sono l'occasione per affrontare la natura e il valore del sapere geografico 'non accademico', non incasellato nelle strette categorie tradizionali della disciplina. 
Affrontando il tema dei cartografi di montagna, Quaini osserva che dalla metà dell'Ottocento i saperi geografici sono stati incanalati in due diversi percorsi divergenti, la "geometrizzazione e progressiva astrazione dello spazio di rappresentazione del territorio" di pretesa scientificità, e il "recupero mediante altri linguaggi dei contenuti dello spazio vissuto e percepito (il paesaggio concreto e sensibile) e delle pratiche e saperi ambientali" espunti dalla conoscenza accademica (ivi, 458).

In questo senso, Verne è letto come l'espressione di una epistemologia dello spazio ottocentesca condivisa tra scienza e letteratura, di "un filo che collega la letteratura fantastica del primo Ottocento, contemporanea e collegata alle grandi spedizioni scientifiche di Humboldt e Chamisso, le utopie fourieriste e sansimoniane, i socialismi anarchici; [...] anzi, più che un filo c'è [...] un ordito e una trama molto fitti" (QUAINI 1995, 65); e varie opere letterarie possono essere considerate come rappresentanti di saperi geografici reali, validi seppur al di fuori degli schemi della geografia 'pre-scientifica' sette-ottocentesca o 'ufficiale' otto-novecentesca, e ad essa collegati in un continuo scambio reciproco di nozioni e di credenze (su queste riflessioni si vedano anche Gambi 1973, 3-37; Quaini 1978, 9-26; SCARAmellini 1985). Non a caso molti dei 'padri nobili' di discipline scientifiche, come Humboldt, sono stati autori di opere letterarie odeporiche, così come la produzione di Calvino ha "una continuità e uno spessore teorico tali da renderla ineludibile anche per il geografo" (Quaini 2008, 87). Per questo motivo una nuova prospettiva di ricerca può e deve essere indirizzata a riannodare questo 'filo spezzato',

adottando una prospettiva più storico-analitica, [per cominciare] a indagare concretamente (per esempio con metodo biografico e di contestualizzazione analitica) gli spazi di transizione fra i diversi linguaggi che in questa fase ancora molto fluida appartengono al sapere geografico: linguaggi cartografici, topografici, artistici, descrittivi, storici, e narrativi (QUAINI 2014, 460),

evitando dicotomie tradizionali come quella tra vedutismo artistico e cartografia scientifica, o tra saperi popolari/letterari e conoscenze disciplinari, ed evidenziandone invece i fattori di osmosi. 
Per concludere, ci si vuole soffermare su un ulteriore problema sollevato da Quaini, ovvero la produzione testuale geografica come forma letteraria. Si tratta di un tema già ampiamente affrontato in ambito antropologico - si pensi alle riflessioni di Clifford Geertz (1987) sulle analogie e differenze tra racconto antropologico demologico scientifico e letteratura realista - che Quaini ripropone alle scienze geografiche, sia nella prassi (producendo una monografia, La mongolfiera di Humbolt - QUAINI 2002 -, contemporaneamente opera letteraria e denso trattato teorico) sia esortando alla nascita di un nuovo genere letterario, il "romanzo geografico". Tale romanzo, "centrato su questioni relative allo spazio geografico e alla sua esplorazione", sarebbe da differenziarsi da quello storico per "l'importanza che vi hanno i luoghi e la loro rappresentazione". Una volta riannodato il filo trasformando un'accurata ricostruzione geografico-storica in narrativa,

vista la quasi coincidenza fra il romanzo e l'indagine scientifica, ci si potrebbe anche chiedere quale sia la differenza fra i due generi. [...] Una restituzione che non può essere paragonata a un processo meccanico e oggettivo. Per me il confine fra i due stili è molto più labile e incerto di quanto può sembrare a prima vista. In fondo, come la storia anche nella prospettiva più scientifica può inclinare al romanzo, anche il romanzo può rendere la storia e il paesaggio con più verità dell'analisi scientifica (QUAINI 2015b, 141-142).

\section{Riferimenti bibliografici}

Anglani P. (2000), "I parchi letterari: nuova forma di organizzazione dello spazio e incentivo allo sviluppo", Bollettino della Società Geografica Italiana, vol. 12, n. 5, pp. 537-539.

Brosseau M. (2017), "In, of, out, with, and through: new perspectives in literary geography”, in Tally R.T. (a cura di), The Routledge Handbook of literature and space, Routledge, London, pp. 9-27.

Fanfani D., Magnaghi A. (2010), "Il parco agricolo, un nuovo strumento per la pianificazione del territorio aperto", in IDD. (a cura di), Patto città campagna. Un progetto di bioregione urbana per la Toscana centrale, Alinea, Firenze, pp. $15-24$.

Fonnesu I., Rombai L. (2004), Letteratura e paesaggio in Toscana. Da Pratesi a Cassola, Italia Nostra, Firenze. 
Gabellieri N. (2019), Geografia letteraria dei paesaggi marginali. La Toscana rurale in Carlo Cassola, All'Insegna del Giglio, Firenze.

Gambi L. (1973), Una geografia per la storia, Einaudi, Torino.

Gavinelli D. (2007), "Geografia e letteratura”, in ID., CAsari M. (a cura di), La letteratura contemporanea nella didattica della geografia e della storia, Cuem, Milano, pp. 5-14.

Gavinelli D., Zanolin G. (2019), Geografia del turismo contemporaneo, Carocci, Roma.

GeERTz C. (1987), Interpretazione di culture, Il Mulino, Bologna.

LANDo F. (1993), Fatto e finzione. Geografia e letteratura, EtasLibri, Milano.

LÉvy B. (2006), "Géographie et littérature: une synthèse historique", Le Globe, n. 146, pp. 25-52.

Marengo M. (2016), Geografia e letteratura. Piccolo manuale d'uso, Pàtron, Bologna.

Moreno D., Guido M.A., Montanari C.A. (2005), "L'approccio storico-archeologico alla copertura vegetale: il contributo dell'archeologia ambientale e dell'ecologia storica", in CANEvA G. (a cura di), La biologia vegetale per $i$ beni culturali, Nardini, Firenze, vol. II, pp. 463-498.

Moreno D., Quaini M. (2016), “Applicazioni della ricerca per il nuovo ruolo di Case Lovara”, in Gabellieri N., Pescini V. (a cura di), Biografia di un paesaggio rurale. Storia, geografia e archeologia ambientale per la riqualificazione di Case Lovara (Promontorio del Mesco - La Spezia), Oltre Edizioni, Sestri Levante, pp. 211-214.

Moreno D., Quaini M., Traldi C. (2016), "Storia di una ricerca: persone, enti e finanziamenti”, in IDD. (a cura di), Dal parco letterario' al parco produttivo, Oltre Edizioni, Sestri Levante, pp. 4-18.

Persi P., DAi Prà E. (2001), 'L'aiuola che ci fa...'. Una geografia per i parchi letterari, Università degli Studi di Urbino, Urbino.

Quaini M. (1978), Dopo la geografia, Espresso Strumenti, a cura di U. Eco, Farigliano.

QuAini M. (1994), "Le parole della geografia. Note in margine ad un dizionario critico della geografia", Notiziario del Centro Italiano per gli Studi StoricoGeografici, vol. 2, n. 1, pp. 22-25.

QuAini M. (1995), “Apparizioni ed eclissi del geografo nell’opera di Jules Verne. Ovvero quando la geografia da 'sogno della scienza' diventa 'scienza dei sogni'”, in Cerreti C. (a cura di), Colonie africane e cultura italiana fra Ottocento e Novecento, Atti dell'incontro di studio, Istituto Italo-africano (Roma, 20 Maggio 1994), CISU, Roma, pp. 49-66

Quaini M. (1996), "Il pellegrinaggio a Waterloo. Una riflessione sui metodi della storia delle geografia", Notiziario del Centro Italiano per gli Studi Storico-Geografici, vol. 4, n. 1, pp. 9-12.

Quaini M. (2002), La mongolfiera di Humboldt. Dialoghi sulla geografia ovvero sul piacere di cercare sulla luna la scienza che non c’è, Diabasis, Reggio Emilia.

Quaini M. (2003), "Costruire Geostorie. Un programma di ricerca per i giovani geografi”, Geostorie. Bollettino e notiziario del Centro Italiano per gli Studi Storico-Geografici, vol. 11, n. 1, pp. 3-15. 
Quaini M. (2006a), L'ombra del paesaggio. L'orizzonte di un'utopia conviviale, Diabasis, Reggio Emilia.

Quaini M. (2006b), "Quale paesaggio per la Liguria del nuovo millennio? Riflessioni in margine a paesaggo e 'geografia culturale", in VARANI N. (a cura di), La Liguria, dal mondo mediterraneo ai nuovi mondi, Brigati, Genova, pp. 481-504.

Quaini M. (2006c), "Tra Sette e Ottocento: il viaggio e il nuovo paradigma della geografia”, in Rossi L., Раротті D. (a cura di), Alla fine del viaggio, Diabasis, Reggio Emilia, pp. 32-46.

Quaini M. (2007), "Introduzione”, in Quaini M., Rossi L. (a cura di), Cartografi in Liguria (secoli XVI-XIX), Brigati, Genova, pp. 4-18.

QuAINI M. (2008), "Poiché niente di quello che la storia sedimenta va perduto", Quaderni Storici, vol. 127, n. 1, pp. 55-108.

Quaini M. (2009a), "La Rocca di Newton e lo sguardo rivoluzionario di Goethe. Sulle condizioni per una nuova storia della geografia italiana”, Geostorie. Bollettino e notiziario del Centro Italiano per gli Studi Storico-Geografici, vol. 18, n. 3, pp. 263-271.

Quaini M. (2009b), "Paesaggi della nostalgia? No grazie", in Rapporto annuale 2009. I paesaggi italiani. Fra nostalgia e trasformazione, Società Geografica Italiana, Roma, pp. 9-19.

Quaini M. (2009c), "Sciascia e Calvino. Il paesaggio e la storia", Il Giannone, vol. 7, n. 13-14, pp. 287-299.

QuAini M. (2010), "Il tempo e lo spazio della collina e della montagna mediterranea”, in Rossi L., Cerretti L.E. (a cura di), Mediterranei, Diabasis, Reggio Emilia, pp. 214-228.

Quaini M. (2011), "Prefazione", in Italiano F., Mastronunzio M. (a cura di), Geopoetiche. Studi di geografia e letteratura, Unicopli, Milano, pp. 7-10.

Quaini M. (2013), "Utopie paesaggistiche: dal paesaggio-angelo al paesaggiospaventapasseri", in Polito P., Roncaccia A. (a cura di), "Entre espace et paysage. Pour une approche interdisciplinaire", Etudes de Lettres, n. 1-2, pp. 293-302.

Quaini M. (2014a), "Le tre vie del paesaggio e il 'ritorno del geografico", in Bonini G., Visentin C. (a cura di), Paesaggi in trasformazione. Teorie e pratiche della ricerca a cinquant'anni dalla Storia del paesaggio agrario italiano di Emilio Sereni, Compositori, Bologna, pp. 57-63.

Quaini M. (2014b), "Nello spirito della Convenzione Europea: una rete di Osservatori locali del paesaggio per creare cittadinanza attiva", in QuAINI M., Gemignani C.A. (a cura di), Cantiere paesaggio. Materiali per la costituzione di osservatori locali, Franco Angeli, Milano, pp. 9-33.

QuAini M. (2014c), "Un grande laboratorio geografico: la montagna alpina fra Sette e Ottocento. Il ruolo della topografia militare", in DAI PRÀ E. (a cura di), Approcci geo-storici e governo del territorio. Vol. 2, Scenari nazionali e internazionali, Franco Angeli, Milano, pp. 451-466.

QuAini M. (2015a), "Leggere il passato per progettare il futuro", in GABELLIERI N., Pescini V. (a cura di), Biografia di un paesaggio rurale. Storia, geografia e archeologia ambientale per la riqualificazione di Case Lovara (Promontorio del Mesco - La Spezia), Oltre Edizioni, Sestri Levante, pp. 209-211. 
QuAini M. (2015b), "Quale geografia per il CISGE? Da castello incantato a castello kafkiano?”, in D’Ascenzo A. (a cura di), Geostoria. Geostorie, CISGE, Roma, pp. 137-149.

Quaini M. (2016a), "Da paese a paesaggio. La lezione mediterranea di Francesco Biamonti”, in Moreno D., Quaini M., Traldi C. (a cura di), Dal parco 'letterario' al parco produttivo, Oltre Edizioni, Sestri Levante, pp. 52-69.

Quaini M. (2016b), "Le paysage est mort, vive le paysage!”, Le Globe. Revue genevoise de géographie, n. 156, pp. 7-18.

Scaramellini G. (1985), "Raffigurazione dello spazio e conoscenza geografica: i resoconti di viaggio", in BIANCHI E. (a cura di), Geografie private. I resoconti di viaggio come letteratura del territorio, Unicopli, Milano, pp. 27-123.

Sereno P. (1981), "La geografia storica in Italia", in Baker A.R.H. (a cura di), Geografia storica. Tendenze e prospettive, Franco Angeli, Milano, pp. 167-187. 